\title{
Expediente
}

\section{Equipe Editorial}

\section{EDITORAS GERAIS}

- $\quad$ Andrea MEDRADO, Universidade Federal Fluminense - UFF, Brasil

- Denise TAVARES, Universidade Federal Fluminense - UFF, Brasil

- Isabella REGA, Bournemouth University, Reino Unido

\section{Editoras da SEÇão TEMÁtica}

- Renata REZENDE, Universidade Federal Fluminense - UFF, Brasil

- Denise Cristina Ayres GOMES, Universidade Federal do Maranhão - UFMA, Brasil

\section{EDITORES ASSISTENTES}

- $\quad$ Davi de Menezes REBOUÇAS - Universidade Federal Fluminense - UFF, Brasil

- $\quad$ Domingos Alves de ALMEIDA - Universidade Federal Fluminense - UFF, Brasil

- $\quad$ Fernanda Angelo COSTANTINO - Universidade Federal Fluminense - UFF, Brasil

- $\quad$ Letycia Gomes NASCIMENTO - Universidade Federal Fluminense - UFF, Brasil

- Pedro Henrique Conceição dos SANTOS - Universidade Federal Fluminense - UFF, Brasil

\section{CAPA}

- Renata REZENDE, Universidade Federal Fluminense - UFF, Brasil (com projeto gráfico de Vitor Silva CHAGAS - Universidade Federal Fluminense - UFF, Brasil)

\section{Consellho Científico}

- Aimée VEGA-MONTIEL, Universidad Nacional Autónoma de México - UNAM, México

- $\quad$ Ana Carolina Rocha Pessôa TEMER, Universidade Federal de Goiás - UFGO, Brasil

- Carlos Alberto ZANOTTI, Pontifícia Universidade Católica de Campinas - PUC, Brasil

- Christina Ferraz MUSSE, Universidade Federal de Juiz de Fora - UFJF, Brasil

- Clementina Galera CASQUET, Universidad de Extremadura, Espanha

- Dênis de MORAES, Universidade Federal Fluminense - UFF, Brasil

- $\quad$ Edson DALMONTE, Universidade Federal da Bahia - UFBA, Brasil

- $\quad$ Francisco KARAM, Universidade Federal de Santa Catarina - UFSC, Brasil

- Gabriela Borges Martins CARAVELA, Universidade Federal de Juiz de Fora - UFJF, Brasil 
- Gabriel KAPLÚN, Universidad de la República Uruguay, Uruguai

- Helena SOUSA, Universidade do Minho, Portugal

- Joseph STRAUBHAAR, University of Texas at Austin, Estados Unidos da América do Norte

- Liriam SPONHOLZ, Department of Media and Communication, Alemanha

- Lucília de S. ROMÃO, Universidade de São Paulo - USP, Brasil

- Luis ALBORNOZ, Universidad Carlos III de Madrid, Espanha

- Marcius FREIRE, Universidade Estadual de Campinas - UNICAMP, Brasil

- Marcos PALÁCIO, Universidade da Beira Interior - UBI, Portugal

- Maria Aparecida BACCEGA, Escola Superior de Propaganda e Marketing - ESPM, Brasil

- Marta Martín LLAGUNO, Universidad de Alicante - UA, Espanha

- Massimo CANEVACCI, Università degli Studi di Roma La Sapienza, Itália

- Muniz SODRÉ, Universidade Federal do Rio de Janeiro - UFRJ, Brasil

- Nelson ZAGALO, Universidade de Aveiro, Portugal

- Nilda A. JACKS, Universidade Federal do Rio Grande do Sul - UFRGS, Brasil

- Raquel PAIVA, Universidade Federal do Rio de Janeiro - UFRJ, Brasil

- Salvatore SCIFO, Bournemouth University, Reino Unido

- Tanja DREHER, University of New South Wales, Sydney, Austrália

\section{Pareceristas desta edicão}

- Adilson Vaz CABRAL FILHO, Universidade Federal Fluminense - PPGMC/UFF, Niterói, Brasil

- Ana Amélia ERTHAL, Escola Superior de Propaganda e Marketing - ESPM, Rio de Janeiro, Brasil

- Bruno FUSER, Universidade Federal de Juiz de Fora - FACOM/ UFJF, Juíz de Fora, Brasil

- Cláudia Regina LAHNI, Universidade Federal de Juiz de Fora - UFJF, Juiz de Fora, Brasil

- Danielle Ramos BRASILIENSE, Universidade Federal Fluminense - PPCULT/UFF, Niterói, Brasil

- Denise Cristina Ayres GOMES, Universidade Federal do Maranhão - PPGCom/UFMA, Imperatriz, Brasil

- Denise da Costa Oliveira SIQUEIRA, Universidade Estadual do Rio de Janeiro - UERJ, Rio de Janeiro, Brasil

- Diego Santos Vieira de JESUS, Escola Superior de Propaganda e Marketing - ESPM, Rio de Janeiro, Brasil

- Dimas KUNSCH, Universidade Metodista de São Paulo - Umesp, São Paulo, Brasil 
- Eula CABRAL, Fundação Casa de Rui Barbosa - Programa de Pós-Graduação em Memória e Acervos, Rio de Janeiro, Brasil

- $\quad$ Erly VIEIRA, Universidade Federal do Espírito Santo - UFES, Vitória, Brasil

- $\quad$ Fernanda CARRERA, Universidade Federal do Rio Grande do Norte - UFRN, Natal, Brasil

- Fernanda ABREU, Universidade Federal Fluminense - UFF, Niterói, Brasil.

- $\quad$ Fernando GONÇALVES, Universidade Federal do Rio de Janeiro - UFRJ, Rio de Janeiro, Brasil

- Gabriela ALVES, Universidade Federal do Espírito Santo - UFES, Vitória, Brasil

- $\quad$ Guilherme ATEM, Universidade Federal Fluminense - UFF, Niterói, Brasil

- José MARTINUZZO, Universidade Federal do Espírito Santo - UFES, Vitória, Brasil

- Juliana DORETTO, FAM Centro Universitario - FAM, Americana, SP, Brasil

- $\quad$ Laura BEDRAN, Universidade Federal Fluminense - UFF, Niterói, Brasil

- Leticia MATHEUS, Universidade Estadual do Rio de Janeiro - UERJ, Rio de Janeiro, Brasil

- $\quad$ Lucas FONSECA, Universidade Federal do Amazonas - UFAM, Parintins, Brasil

- Marcio de Souza CASTILHO, Universidade Federal Fluminense - UFF, Niterói, Brasil

- $\quad$ Mateus Yuri PASSOS, Universidade Metodista de São Paulo - Umesp, São Paulo, Brasil

- Mariana Ferreira POMBO, Universidade Federal do Rio de Janeiro - UFRJ, Rio de Janeiro, Brasil

- Marta MAIA, Universidade Federal de Ouro Preto, - UFOP, Ouro Preto/MG, Brasil

- $\quad$ Miguel Luiz CONTANI, Universidade Estadual de Londrina - UEL, Paraná, Brasil

- Marina CAMINHA, Consejo Latino-Americano de Ciencias Sociales - CLACSO, Argentina

- Mohammed ELHAJJI, Universidade Federal do Rio de Janeiro - UFRJ, Rio de Janeiro, Brasil

- $\quad$ Pablo Nabarrete BASTOS, Universidade Federal Fluminense - PPGMC/UFF, Niterói, Brasil

- Paulo Cesar CASTRO, Universidade Federal do Rio de Janeiro - UFRJ, Rio de Janeiro, Brasil

- $\quad$ Rafael FORTES, Universidade Federal do Rio de Janeiro - UFRJ, Rio de Janeiro, Brasil

- $\quad$ Renata REZENDE, Universidade Federal Fluminense - PPGMC/UFF, Niterói, Brasil

- $\quad$ Simone do VALE, Faculdades Integradas Hélio Alonso - FACHA, Rio de Janeiro, Brasil

- $\quad$ Sonia AGUIAR, Universidade Federal de Sergipe - UFS, Aracajú, Brasil

- Vitor BRAGA, Universidade Federal de Sergipe - UFS, Aracajú, Brasil

- Walcea Barreto ALVES, Universidade Federal Fluminense - PPGMC/UFF, Niterói, Brasil

Data da Publicação: 26 de agosto de 2019 